Pacific

Journal of

Mathematics

\title{
COHOMOLOGY AND EXTENSIONS OF BRACES
}

VICTORIA LEBED AND LEANDRO VENDRAMIN

Volume 284 No. 1

September 2016 


\title{
COHOMOLOGY AND EXTENSIONS OF BRACES
}

\author{
VICTORIA LEBED AND LEANDRO VENDRAMIN
}

\begin{abstract}
Braces and linear cycle sets are algebraic structures playing a major role in the classification of involutive set-theoretic solutions to the Yang-Baxter equation. This paper introduces two versions of their (co)homology theories. These theories mix the Harrison (co)homology for the abelian group structure and the (co)homology theory for general cycle sets, developed earlier by the authors. Different classes of brace extensions are completely classified in terms of second cohomology groups.
\end{abstract}

\section{Introduction}

A (left) brace is an abelian group $(A,+)$ with an additional group operation o such that for all $a, b, c \in A$, the following compatibility condition holds:

$$
a \circ(b+c)+a=a \circ b+a \circ c .
$$

The two group structures necessarily share the same neutral element, denoted by 0 . Braces, in a slightly different but equivalent form, were introduced by Rump [2007]; the definition above goes back to Cedó, Jespers, and Okniński [Cedó et al. 2014]. To get a feeling of what braces look like, and to convince oneself that they are not as rare in practice as one might think, the reader is referred to Bachiller's classification of braces of order $p^{3}$ [2015a]. The growing interest into these structures is due to a number of reasons. First, braces generalize radical rings. Second, Catino and Rizzo [2009] and Catino, Colazzo, and Stefanelli [Catino et al. 2015; 2016] unveiled the role of an $F$-linear version of this notion into the classification problem for regular subgroups of affine groups over a field $F$. Third, braces are enriched cycle sets, and are therefore important in the study of set-theoretic solutions to the Yang-Baxter equation (YBE), as we now recall.

A cycle set, as defined by Rump [2005], is a set $X$ with a binary operation . having bijective left translations $X \rightarrow X, a \mapsto b \cdot a$, and satisfying the relation

$$
(a \cdot b) \cdot(a \cdot c)=(b \cdot a) \cdot(b \cdot c) .
$$

MSC2010: 20E22, 20N02, 55N35, 16T25.

Keywords: brace, cycle set, Yang-Baxter equation, extension, cohomology. 
Rump showed that nondegenerate cycle sets (i.e., with invertible squaring map $a \mapsto a \cdot a)$ are in bijection with nondegenerate involutive set-theoretic solutions to the Yang-Baxter equation. Such solutions form a combinatorially rich class of structures, connected with many other domains of algebra: semigroups of I-type, Bieberbach groups, Hopf algebras, Garside groups, etc. The cycle set approach turned out to be extremely fruitful for elucidating the structure of such solutions and obtaining classification results (see, for instance, [Cedó et al. 2010a; 2014; Chouraqui 2010; Dehornoy 2015; Gateva-Ivanova 2015; Gateva-Ivanova and Majid 2008; Gateva-Ivanova and Van den Bergh 1998; Jespers and Okniński 2005; Rump 2007; 2008; 2014; Smoktunowicz 2015a; 2015b; Vendramin 2016] and references therein). In spite of the intensive ongoing research on cycle sets, their structure is still far from being completely understood. This can be illustrated by numerous conjectures and open questions in the area, many of which were formulated by Gateva-Ivanova and Cameron [Gateva-Ivanova 2004; Gateva-Ivanova and Cameron 2012] and by Cedó, Jespers, and del Río [Cedó et al. 2010b].

Etingof, Schedler, and Soloviev [Etingof et al. 1999] initiated the study of the structure group of a solution to the YBE - and in particular of a cycle set. These ideas were further explored in [Lu et al. 2000; Soloviev 2000] for noninvolutive solutions. Concretely, the structure group $G_{(X, \cdot)}$ of a cycle set $(X, \cdot)$ is the free group on the set $X$, modulo the relations

$$
(a \cdot b) a=(b \cdot a) b
$$

for all $a, b \in X{ }^{1}$ In [Etingof et al. 1999], the structure group of a nondegenerate cycle set $(X, \cdot)$ was shown to be isomorphic, as a set, to the free abelian group $\mathbb{Z}^{(X)}$ on $X$; see also [Lebed and Vendramin 2015] for an explicit graphical form of this isomorphism. The group $G_{(X, \cdot)}$ thus carries a second, abelian, group structure the one pulled back from $\mathbb{Z}^{(X)}$ - and becomes a brace. Moreover, $G_{(X, \cdot)}$ inherits a cycle set structure from $X$, and yields a key example of the following notion. A linear cycle set is a cycle set $(A, \cdot)$ with an abelian group operation + satisfying, for all $a, b, c \in A$, the compatibility conditions

$$
\begin{aligned}
& a \cdot(b+c)=a \cdot b+a \cdot c, \\
& (a+b) \cdot c=(a \cdot b) \cdot(a \cdot c) .
\end{aligned}
$$

This structure also goes back to Rump [2007], who showed it to be equivalent to the brace structure, via the relation

$$
a \cdot b=a^{-1} \circ(a+b) .
$$

\footnotetext{
${ }^{1}$ Some authors prefer an alternative relation $a(a \cdot b)=b(b \cdot a)$, which defines an isomorphic group.
} 
Understanding structure groups and certain classes of their quotients is often regarded as a reasonable first step towards understanding cycle sets. Even better: Bachiller, Cedó, and Jespers [Bachiller et al. 2015a] recently reduced the classification problem for cycle sets to that for braces. This explains the growing interest in braces and linear cycle sets. As pointed out by Bachiller, Cedó, Jespers, and Okniński [Bachiller et al. 2015b], an extension theory for braces would be crucial for classification purposes, as well as for elaborating new examples. This served as motivation for our paper.

Lebed and Vendramin [2015] developed a cohomology theory for general cycle sets, in which second cohomology groups were given particular attention: they were shown to encode central cycle set extensions. Here we propose homology and cohomology theories for linear cycle sets, and thus for braces. As usual, central linear cycle set extensions turn out to be classified by the second cohomology groups.

For pedagogical reasons, we first study extensions that are trivial on the level of abelian groups, together with a corresponding (co)homology theory (Sections 2-3). Such extensions are still of interest, since it is often the cycle set operation that is the most significant part of the linear cycle set structure (as in the example of structure groups). On the other hand, they are technically much easier to handle than the general extensions (Sections 4-5). We therefore found it instructive to present this "reduced" case before the general one.

When finishing this paper, we learned that an analogous extension theory was independently developed by Bachiller [2015b], using the language of braces. Some fragments of it in the $F$-linear setting also appeared in the work of Catino, Colazzo, and Stefanelli [2015]. An alternative approach to extensions was suggested earlier by Ben David and Ginosar [2016]. Concretely, they studied the lifting problem for bijective 1-cocycles - which is yet another avatar of braces. Their work was translated into the language of braces by Bachiller [2015a]. Our choice of the linear cycle set language leads to more transparent constructions. Moreover, it made possible the development of a full cohomology theory extending the degree 2 constructions motivated by the extension analysis. Such a theory was missing in all the previous approaches.

\section{Reduced linear cycle set cohomology}

From now on we work with linear cycle sets (LCS). As explained in the introduction, all constructions and results can be directly translated into the language of braces. We will perform this translation for major results only.

Take an LCS $(A, \cdot,+)$ and an abelian group $\Gamma$. For $n>0$, let $R C_{n}(A ; \Gamma)$ denote the abelian group $\Gamma \otimes_{\mathbb{Z}} \mathbb{Z} A^{\times n} \simeq \Gamma^{\left(A^{\times n}\right)}$, modulo the linearity relation

$$
\gamma\left(a_{1}, \ldots, a_{n-1}, a_{n}+a_{n}^{\prime}\right)=\gamma\left(a_{1}, \ldots, a_{n-1}, a_{n}\right)+\gamma\left(a_{1}, \ldots, a_{n-1}, a_{n}^{\prime}\right)
$$


for the last copy of $A$. Denote by $R C_{n}^{\mathrm{D}}(A ; \Gamma)$ the abelian subgroup of $R C_{n}(A ; \Gamma)$ generated by the degenerate $n$-tuples, i.e., $\gamma\left(a_{1}, \ldots, a_{n}\right)$ with $a_{i}=0$ for some $1 \leqslant i \leqslant n$. Consider also the quotient $R C_{n}^{\mathrm{N}}(A ; \Gamma)=R C_{n}(A ; \Gamma) / R C_{n}^{\mathrm{D}}(A ; \Gamma)$. Further, define the maps $\partial_{n}: \Gamma A^{\times n} \rightarrow \Gamma A^{\times(n-1)}, n>1$, as the linearizations of

$$
\begin{aligned}
\partial_{n}\left(a_{1}, \ldots, a_{n}\right)=( & \left.a_{1} \cdot a_{2}, \ldots, a_{1} \cdot a_{n}\right) \\
& +\sum_{i=1}^{n-2}(-1)^{i}\left(a_{1}, \ldots, a_{i}+a_{i+1}, \ldots, a_{n}\right) \\
& +(-1)^{n-1}\left(a_{1}, \ldots, a_{n-2}, a_{n}\right) .
\end{aligned}
$$

Complete this family of maps by $\partial_{1}=0$. Dually, for $n>0$, let $R C^{n}(A ; \Gamma)$ denote the set of maps $f: A^{\times n} \rightarrow \Gamma$ linear in the last coordinate:

$$
f\left(a_{1}, \ldots, a_{n-1}, a_{n}+a_{n}^{\prime}\right)=f\left(a_{1}, \ldots, a_{n-1}, a_{n}\right)+f\left(a_{1}, \ldots, a_{n-1}, a_{n}^{\prime}\right),
$$

and let $R C_{\mathrm{N}}^{n}(A ; \Gamma) \subset R C^{n}(A ; \Gamma)$ comprise the maps vanishing on all degenerate $n$-tuples. Define the maps $\partial^{n}: \operatorname{Fun}\left(A^{\times n}, \Gamma\right) \rightarrow \operatorname{Fun}\left(A^{\times(n+1)}, \Gamma\right), n \geqslant 1$, by

$$
\begin{aligned}
\left(\partial^{n} f\right)\left(a_{1}, \ldots, a_{n+1}\right)=f & \left(a_{1} \cdot a_{2}, \ldots, a_{1} \cdot a_{n+1}\right) \\
& +\sum_{i=1}^{n-1}(-1)^{i} f\left(a_{1}, \ldots, a_{i}+a_{i+1}, \ldots, a_{n+1}\right) \\
& +(-1)^{n} f\left(a_{1}, \ldots, a_{n-1}, a_{n+1}\right) .
\end{aligned}
$$

These formulas resemble the group (co)homology construction for $(A,+)$. We will now show that they indeed define a (co)homology theory.

Proposition 2.1. Let $(A, \cdot,+)$ be a linear cycle set and $\Gamma$ be an abelian group.

(1) The maps $\partial_{\text {. above }}$

- square to zero: $\partial_{n-1} \partial_{n}=0$ for all $n>1$;

- induce maps $R C_{n}(A ; \Gamma) \rightarrow R C_{n-1}(A ; \Gamma)$;

- and further restrict to maps $R C_{n}^{\mathrm{D}}(A ; \Gamma) \rightarrow R C_{n-1}^{\mathrm{D}}(A ; \Gamma)$.

(2) The maps $\partial^{\bullet}$ above

- square to zero: $\partial^{n+1} \partial^{n}=0$ for all $n \geqslant 1$;

- restrict to maps $R C^{n}(A ; \Gamma) \rightarrow R C^{n+1}(A ; \Gamma)$;

- and further restrict to maps $R C_{\mathrm{N}}^{n}(A ; \Gamma) \rightarrow R C_{\mathrm{N}}^{n+1}(A ; \Gamma)$.

The induced or restricted maps from the proposition will be abusively denoted by the same symbols $\partial_{\bullet}, \partial^{\bullet}$. In the proof we shall need the special properties of the zero element of an LCS.

Lemma 2.2. In any LCS $A$, the relations $a \cdot 0=0$ and $0 \cdot a=a$ hold for all $a \in A$. 
Proof. By the LCS axioms, one has $a \cdot 0=a \cdot(0+0)=a \cdot 0+a \cdot 0$ and hence $a \cdot 0=0$. Similarly, $0 \cdot a=(0+0) \cdot a=(0 \cdot 0) \cdot(0 \cdot a)=0 \cdot(0 \cdot a)$, and the relation $a=0 \cdot a$ follows by canceling out 0 (recall that the left translation $0 \cdot-$ is bijective).

Proof of Proposition 2.1. We treat only the homological statements here; they imply the cohomological ones by duality.

The maps $\partial_{n}$ can be presented as signed sums $\partial_{n}=\sum_{i=0}^{n-1}(-1)^{i} \partial_{n ; i}$, where

$$
\begin{aligned}
\partial_{n ; 0}\left(a_{1}, \ldots, a_{n}\right) & =\left(a_{1} \cdot a_{2}, \ldots, a_{1} \cdot a_{n}\right), \\
\partial_{n ; i}\left(a_{1}, \ldots, a_{n}\right) & =\left(a_{1}, \ldots, a_{i}+a_{i+1}, \ldots, a_{n}\right), \quad 1 \leqslant i \leqslant n-2, \\
\partial_{n ; n-1}\left(a_{1}, \ldots, a_{n}\right) & =\left(a_{1}, \ldots, a_{n-2}, a_{n}\right) .
\end{aligned}
$$

The relation $\partial_{n-1} \partial_{n}=0$ then classically reduces to the "almost commutativity" $\partial_{n-1 ; j} \partial_{n ; i}=\partial_{n-1 ; i} \partial_{n ; j+1}$ for all $i \leqslant j$. In the case $i>0$ this latter relation is either tautological, or follows from the associativity of + . For $i=0<j$, it follows from the left distributivity (1-3) for $A$. For $i=0=j$, it is a consequence of the second LCS relation (1-4) for $A$.

Further, using the linearity (1-3) of the left translations $a_{n} \mapsto a_{1} \cdot a_{n}$, one sees that when applied to expressions of type

$$
\left(\ldots, a_{n-1}, a_{n}+a_{n}^{\prime}\right)-\left(\ldots, a_{n-1}, a_{n}\right)-\left(\ldots, a_{n-1}, a_{n}^{\prime}\right),
$$

all the maps $\partial_{n ; i}$ yield expressions of the same type. Hence their signed sums $\partial_{\bullet}$ induce a differential on $R C_{\bullet}$. The possibility to further restrict to $R C_{\bullet}^{\mathrm{D}}$ is guaranteed by Lemma 2.2 .

Proposition 2.1 legitimizes the following definition:

Definition 2.3. The reduced (resp., normalized) cycles, boundaries, and homology groups of a linear cycle set $(A, \cdot,+)$ with coefficients in an abelian group $\Gamma$ are those of the chain complex $\left(R C_{\bullet}(A ; \Gamma), \partial_{\bullet}\right)\left(\operatorname{resp} .,\left(R C_{\bullet}^{\mathrm{N}}(A ; \Gamma), \partial_{\bullet}\right)\right)$ above. Dually, the reduced (resp., normalized) cocycles, coboundaries, and cohomology groups of $(A, \cdot,+)$ are those of the complex $\left(R C^{\bullet}(A ; \Gamma), \partial^{\bullet}\right)\left(\operatorname{resp} .,\left(R C_{\mathrm{N}}^{\bullet}(A ; \Gamma), \partial^{\bullet}\right)\right)$. We use the usual notation for these groups: $R Q_{n}(A ; \Gamma), R Q_{n}^{\mathrm{N}}(A ; \Gamma), R Q^{n}(A ; \Gamma)$, $R Q_{\mathrm{N}}^{n}(A ; \Gamma)$, where $Q$ is one of the letters $Z, B$, or $H$.

Remark 2.4. We actually showed that our (co)homology constructions can be refined into (co)simplicial ones in the proof of Proposition 2.1.

Example 2.5. Recall from the introduction that for a nondegenerate cycle set $(X, \cdot)$, the free abelian group $\left(\mathbb{Z}^{(X)},+\right)$ can be seen as a linear cycle set, with the cycle set operation induced from $\cdot$. In this case $R C_{1}\left(\mathbb{Z}^{(X)} ; \Gamma\right)$ is simply the abelian group $\Gamma \otimes_{\mathbb{Z}} \mathbb{Z}^{(X)}=\Gamma^{(X)}$, and for $a_{1}, a_{2} \in \mathbb{Z}^{(X)}$ one calculates $\partial_{1}\left(a_{1}, a_{2}\right)=a_{1} \cdot a_{2}-a_{2}$. Standard arguments from LCS theory then yield

$$
R H_{1}\left(\mathbb{Z}^{(X)} ; \Gamma\right) \cong \Gamma^{(\operatorname{Orb}(X))},
$$


where $\operatorname{Orb}(X)$ is the set of orbits of $X$, i.e., classes for the equivalence relation generated by $a_{1} \cdot a_{2} \sim a_{2}$ for all $a_{1}, a_{2} \in X$. Similarly, one calculates the first reduced cohomology group:

$$
R H^{1}\left(\mathbb{Z}^{(X)} ; \Gamma\right) \cong \operatorname{Fun}(\operatorname{Orb}(X), \Gamma) .
$$

We finish with a comparison between the (co)homology of an LCS $(A, \cdot,+)$ and the (co)homology of its underlying cycle set $(A, \cdot)$, as defined in [Lebed and Vendramin 2015]. Recall that the homology $H_{n}^{\mathrm{CS}}(A ; \Gamma)$ of $(A, \cdot)$ is computed by the complex $\left(\Gamma^{\left(A^{\times n}\right)}, \partial_{n}^{\mathrm{CS}}\right)$, where

$$
\begin{array}{r}
\partial_{n}^{\mathrm{CS}}\left(a_{1}, \ldots, a_{n}\right)=\sum_{i=1}^{n-1}(-1)^{i-1}\left(\left(a_{i} \cdot a_{1}, \ldots, a_{i} \cdot a_{i-1}, a_{i} \cdot a_{i+1}, \ldots, a_{i} \cdot a_{n}\right)\right. \\
\left.-\left(a_{1}, \ldots, a_{i-1}, a_{i+1}, \ldots, a_{n}\right)\right) .
\end{array}
$$

Dually, the cohomology $H_{\mathrm{CS}}^{n}(A ; \Gamma)$ of $(A, \cdot)$ is computed from the complex

$$
\left(\operatorname{Fun}\left(A^{\times n}, \Gamma\right), \partial_{\mathrm{CS}}^{n}\right), \quad \text { with } \partial_{\mathrm{CS}}^{n} f=f \circ \partial_{n+1}^{\mathrm{CS}} \text {. }
$$

Denoting by $(-1)^{\sigma}$ the sign of the permutation $\sigma$, define $S_{n}: \Gamma^{\left(A^{\times n}\right)} \rightarrow R C_{n}(A ; \Gamma)$ as the composition of the antisymmetrization map

$$
\gamma\left(a_{1}, \ldots, a_{n}\right) \mapsto \sum_{\sigma \in \operatorname{Sym}_{n-1}}(-1)^{\sigma} \gamma\left(a_{\sigma(1)}, \ldots, a_{\sigma(n-1)}, a_{n}\right)
$$

and the obvious projection $\Gamma^{\left(A^{\times n}\right)} \rightarrow R C_{n}(A ; \Gamma)$.

Proposition 2.6. Let $(A, \cdot,+)$ be a linear cycle set and $\Gamma$ be an abelian group. The map $S$ defined above yields a map of chain complexes

$$
S_{n}:\left(\Gamma^{\left(A^{\times n}\right)}, \partial_{n}^{\mathrm{CS}}\right) \rightarrow\left(R C_{n}(A ; \Gamma), \partial_{n}\right) .
$$

Proof. One has to compare the evaluations of the maps $\partial_{n} S_{n}$ and $S_{n-1} \partial_{n}^{\mathrm{CS}}$ on $\gamma\left(a_{1}, \ldots, a_{n}\right)$. For this, it is convenient to use the decomposition

$$
\partial_{n}=\sum_{i=0}^{n-1}(-1)^{i} \partial_{n ; i}
$$

from (2-5)-(2-7). For $0<i<n-1$, the map $\partial_{n ; i} S_{n}$ is zero: in its evaluation, the terms $\pm \gamma\left(\ldots, a_{j}+a_{k}, \ldots\right)$ and $\mp \gamma\left(\ldots, a_{k}+a_{j}, \ldots\right)$, with the sum at the $i$-th 
position, cancel. A careful sign inspection yields

$$
\begin{aligned}
& \partial_{n ; 0} S_{n}\left(\gamma\left(a_{1}, \ldots, a_{n}\right)\right) \\
& \quad=\sum_{i=1}^{n-1}(-1)^{i-1} S_{n-1}\left(\gamma\left(a_{i} \cdot a_{1}, \ldots, a_{i} \cdot a_{i-1}, a_{i} \cdot a_{i+1}, \ldots, a_{i} \cdot a_{n}\right)\right), \\
& \begin{aligned}
& \partial_{n ; n-1} S_{n}\left(\gamma\left(a_{1}, \ldots, a_{n}\right)\right) \\
&= \sum_{i=1}^{n-1}(-1)^{n-1-i} S_{n-1}\left(\gamma\left(a_{1}, \ldots, a_{i-1}, a_{i+1}, \ldots, a_{n}\right)\right),
\end{aligned}
\end{aligned}
$$

hence the maps $\partial_{n} S_{n}$ and $S_{n-1} \partial_{n}^{\mathrm{CS}}$ coincide.

As a consequence, one obtains the dual map

$$
S^{n}:\left(R C^{n}(A ; \Gamma), \partial^{n}\right) \rightarrow\left(\operatorname{Fun}\left(A^{\times n}, \Gamma\right), \partial_{\mathrm{CS}}^{n}\right)
$$

of cochain complexes, and the induced maps in (co)homology.

\section{Cycle-type extensions vs. reduced 2-cocycles}

We now turn to a study of the reduced 2-cocycles of a linear cycle set $(A, \cdot,+)$, i.e., maps $f: A \times A \rightarrow \Gamma$ (where $\Gamma$ is an abelian group) satisfying

$$
\begin{aligned}
& f(a, b+c)=f(a, b)+f(a, c), \\
& f(a+b, c)=f(a \cdot b, a \cdot c)+f(a, c),
\end{aligned}
$$

for all $a, b, c \in A$. The last relation, together with the commutativity of + , yields

$$
f(a \cdot b, a \cdot c)+f(a, c)=f(b \cdot a, b \cdot c)+f(b, c),
$$

implying $\partial_{\mathrm{CS}}^{2}(f)=0$, so our $f$ is necessarily a cocycle of the cycle set $(A, \cdot)$.

Among the reduced 2-cocycles we distinguish the reduced 2-coboundaries

$$
\partial^{1}(\theta)(a, b)=\theta(a \cdot b)-\theta(b),
$$

where the map $\theta: A \rightarrow \Gamma$ is linear.

Example 3.1. Let $A$ and $\Gamma$ be abelian groups. Consider the trivial linear cycle set structure $a \cdot \operatorname{tr} b=b$ over $A$. A map $f: A \times A \rightarrow \Gamma$ is a reduced 2-cocycle of this LCS if and only if $f$ is a bicharacter, in the sense of the bilinearity relations

$$
f(a+b, c)=f(a, c)+f(b, c) \quad \text { and } \quad f(a, b+c)=f(a, b)+f(a, c) .
$$

The reduced 2-coboundaries are all trivial in this case. Thus $R H^{2}(A ; \Gamma)$ is the abelian group of bicharacters of $A$ with values in $\Gamma$. Observe that for the cycle set $\left(A,{ }^{t r}\right)$, all the differentials $\partial_{\mathrm{CS}}^{n}$ vanish. The second cohomology group $H_{\mathrm{CS}}^{2}(A ; \Gamma)$ 
of this cycle set thus comprises all the maps $f: A \times A \rightarrow \Gamma$, and is strictly larger than $R H^{2}(A ; \Gamma)$.

Example 3.2. Let $A=\{0,1,2,3\}=\mathbb{Z} / 4$ be the cyclic group of 4 elements written additively. Then $A$ is a brace with

$$
a \circ b=a+b+2 a b \quad \text { and } \quad a^{-1}=(2 a-1) a .
$$

The corresponding linear cycle set structure on $A$ is given by the operation

$$
a \cdot b=a^{-1} \circ(a+b)=(1+2 a) b,
$$

which is $b$ when one of $a, b$ is even, and $b+2$ otherwise. Take $\Gamma=\{0,1\}=\mathbb{Z} / 2$. For a map $f: \mathbb{Z} / 4 \times \mathbb{Z} / 4 \rightarrow \mathbb{Z} / 2$, relation (3-1) means that $f$ is of the form $f(a, b)=b \psi(a)$ (where the product is taken in $\mathbb{Z} / 2$, and $b$ is reduced modulo 2 ), for some $\psi: \mathbb{Z} / 4 \rightarrow \mathbb{Z} / 2$. Relation (3-2) then translates as

$$
\psi(a+b)=\psi(b+2 a b)+\psi(a) .
$$

The substitution $b=0$ yields $\psi(0)=0$. Analyzing other values of $a$ and $b$, one sees that $\psi(1)$ and $\psi(3)$ can be chosen arbitrarily, and $\psi(2)$ has to equal $\psi(1)+\psi(3)$. The reduced 2-coboundaries are again trivial: a linear map $\theta: \mathbb{Z} / 4 \rightarrow \mathbb{Z} / 2$ is necessarily of the form $\theta(a)=a t$ for some constant $t \in \mathbb{Z} / 2$, yielding

$$
\theta(a \cdot b)=(a \cdot b) t=(1+2 a) b t=b t=\theta(b)
$$

(since $2 a=0$ in $\mathbb{Z} / 2$ ). Summarizing, one gets

$$
R H^{2}(\mathbb{Z} / 4 ; \mathbb{Z} / 2) \simeq \mathbb{Z} / 2 \times \mathbb{Z} / 2 .
$$

Let us now turn to the underlying cycle set $(\mathbb{Z} / 4, \cdot)$. Playing with (3-3), one verifies that its 2 -cocycles are maps $f: \mathbb{Z} / 4 \times \mathbb{Z} / 4 \rightarrow \mathbb{Z} / 2$ verifying 3 linear relations:

$$
\begin{aligned}
f(0,1)+f(0,3) & =0, \\
f(2,1)+f(2,3) & =0, \\
f(1,1)+f(1,3)+f(3,1)+f(3,3) & =0 .
\end{aligned}
$$

Its only nontrivial 2-coboundary is $f(a, b)=a b \bmod 2$. This implies

$$
\begin{aligned}
& Z_{\mathrm{CS}}^{2}(\mathbb{Z} / 4 ; \mathbb{Z} / 2) \simeq(\mathbb{Z} / 2)^{4 \times 4-3}=(\mathbb{Z} / 2)^{13}, \\
& H_{\mathrm{CS}}^{2}(\mathbb{Z} / 4 ; \mathbb{Z} / 2) \simeq(\mathbb{Z} / 2)^{12} .
\end{aligned}
$$

We will now construct extensions of our LCS $A$ by $\Gamma$ out of 2-cocycles, show that any central cycle-type extension is isomorphic to one of this type, and that reduced 2-cocycles, modulo reduced 2-coboundaries, classify such extensions. 
Lemma 3.3. Let $(A, \cdot,+)$ be a linear cycle set, let $\Gamma$ be an abelian group, and let $f: A \times A \rightarrow \Gamma$ be a map. Then the abelian group $\Gamma \oplus A$ with the operation

$$
(\gamma, a) \cdot\left(\gamma^{\prime}, a^{\prime}\right)=\left(\gamma^{\prime}+f\left(a, a^{\prime}\right), a \cdot a^{\prime}\right), \quad \gamma, \gamma^{\prime} \in \Gamma, a, a^{\prime} \in A
$$

is a linear cycle set if and only if $f$ is a reduced 2-cocycle, i.e., $f \in R Z^{2}(A ; \Gamma)$.

Notation 3.4. The LCS from the lemma is denoted by $\Gamma \oplus_{f} A$.

Proof. The left translation invertibility for $\Gamma \oplus_{f} A$ follows from to the same property for $A$. Properties (1-3) and (1-4) are equivalent for $\Gamma \oplus_{f} A$ to, respectively, properties (3-1) and (3-2) from the definition of a 2-cocycle for $f$. The cycle set property (1-2) follows from (1-4) and the commutativity of + .

Lemma 3.3 and the correspondence between linear cycle sets and braces yield the following result.

Lemma 3.5. Let $(A, \circ,+)$ be a brace, let $\Gamma$ be an abelian group, and let $f$ : $A \times A \rightarrow \Gamma$ be a map. Then the abelian group $\Gamma \oplus A$ with the product

$$
(\gamma, a) \circ\left(\gamma^{\prime}, a^{\prime}\right)=\left(\gamma+\gamma^{\prime}+f\left(a, a^{\prime}\right), a \circ a^{\prime}\right), \quad \gamma, \gamma^{\prime} \in \Gamma, a, a^{\prime} \in A,
$$

is a brace if and only if for the corresponding linear cycle set $(A, \cdot,+)$, the map $\bar{f}(a, b)=f(a, a \cdot b)$ is a reduced 2-cocycle.

Before introducing the notion of LCS extensions, we need some preliminary definitions.

Definition 3.6. A morphism between linear cycle sets $A$ and $B$ is a map $\varphi: A \rightarrow B$ preserving the structure, i.e., for all $a, a^{\prime} \in A$ one has $\varphi\left(a+a^{\prime}\right)=\varphi(a)+\varphi\left(a^{\prime}\right)$ and $\varphi\left(a \cdot a^{\prime}\right)=\varphi(a) \cdot \varphi\left(a^{\prime}\right)$. The kernel of $\varphi$ is defined by $\operatorname{Ker} \varphi=\varphi^{-1}(0)$. The notions of the image $\operatorname{Im} \varphi=\varphi(A)$, of a short exact sequence of linear cycle sets, and of linear cycle subsets, are defined in the obvious way. A linear cycle subset $A^{\prime}$ of $A$ is called central if for all $a \in A, a^{\prime} \in A^{\prime}$, one has $a \cdot a^{\prime}=a^{\prime}$ and $a^{\prime} \cdot a=a$.

For a LSC morphism $\varphi: A \rightarrow B, \operatorname{Ker} \varphi$ and $\operatorname{Im} \varphi$ are clearly linear cycle subsets of $A$ and $B$ respectively. Lemma 2.2 can be rephrased by stating that $\{0\}$ is a central linear cycle subset of $A$.

Definition 3.7. A central cycle-type extension of a linear cycle set $(A, \cdot,+)$ by an abelian group $\Gamma$ is the datum of a short exact sequence of linear cycle sets

$$
0 \rightarrow \Gamma \stackrel{\iota}{\rightarrow} E \stackrel{\pi}{\rightarrow} A \rightarrow 0
$$

where $\Gamma$ is endowed with the trivial cycle set structure $\gamma \cdot \gamma^{\prime}=\gamma^{\prime}$, its image $\iota(\Gamma)$ is central in $E$ (in the sense of Definition 3.6), and the short exact sequence of abelian groups underlying (3-4) splits. 
The adjective cycle-type refers here to the fact that our extensions are interesting on the level of the cycle set operation - only, and trivial on the level of the additive operation + , since we require the short exact sequences to linearly split. More general extensions - those taking into account the additive operation as well - are postponed until the next section. Cycle-type extensions are important, for example, for comparing the LCS structures on the structure group of a cycle set before and after a cycle set extension; see the introduction for more detail on structure groups, and [Lebed and Vendramin 2015] for the cycle set extension theory.

The LCS $\Gamma \oplus_{f} A$ from Lemma 3.3 is an extension of $A$ by $\Gamma$ in the obvious way. We now show that this example is essentially exhaustive.

Definition 3.8. Two central cycle-type LCS extensions

$$
\Gamma \stackrel{\iota}{\longmapsto} E \stackrel{\pi}{\rightarrow} A \quad \text { and } \quad \Gamma \stackrel{\iota^{\prime}}{\longmapsto} E^{\prime} \stackrel{\pi^{\prime}}{\rightarrow} A
$$

are called equivalent if there exists an LCS isomorphism $\varphi: E \rightarrow E^{\prime}$ making the following diagram commute:

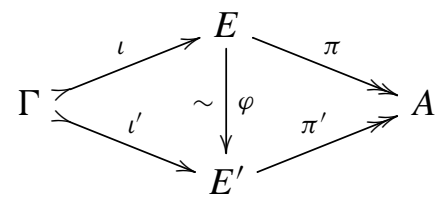

The set of equivalence classes of central cycle-type extensions of $A$ by $\Gamma$ is denoted by $\operatorname{CTExt}(A, \Gamma)$.

Lemma 3.9. Let $\Gamma \stackrel{\iota}{\longmapsto} E \stackrel{\pi}{\rightarrow} A$ be a central cycle-type LCS extension, and $s: A \rightarrow E$ be a linear section of $\pi$. Then the map

$$
\tilde{f}: A \times A \rightarrow E, \quad\left(a, a^{\prime}\right) \mapsto s(a) \cdot s\left(a^{\prime}\right)-s\left(a \cdot a^{\prime}\right)
$$

takes values in $\iota(\Gamma)$ and defines a reduced cocycle $f \in R Z^{2}(A ; \Gamma)$. Extensions $E$ and $\Gamma \oplus_{f} A$ are equivalent. Furthermore, a cocycle $f^{\prime}$ obtained from another section $s^{\prime}$ of $\pi$ is cohomologous to $f$.

Proof. The computation

$$
\pi\left(\tilde{f}\left(a, a^{\prime}\right)\right)=\pi s(a) \cdot \pi s\left(a^{\prime}\right)-\pi s\left(a \cdot a^{\prime}\right)=a \cdot a^{\prime}-a \cdot a^{\prime}=0
$$

yields $\operatorname{Im} \tilde{f} \subseteq \operatorname{Ker} \pi=\operatorname{Im} \iota$ (by the definition of a short exact sequence). Hence the map $f: A \times A \rightarrow \Gamma$ can be defined by the formula $f=\iota^{-1} \tilde{f}$. It remains to check relations (3-1)-(3-2) for this map. The linearity of $s$ and of the left translations 
$t_{b}: a \mapsto b \cdot a$ gives

$$
\begin{aligned}
\tilde{f}(a, b+c) & =s(a) \cdot s(b+c)-s(a \cdot(b+c)) \\
& =s(a) \cdot(s(b)+s(c))-s(a \cdot b+a \cdot c) \\
& =s(a) \cdot s(b)+s(a) \cdot s(c)-s(a \cdot b)-s(a \cdot c)=\tilde{f}(a, b)+\tilde{f}(a, c) .
\end{aligned}
$$

hence $f(a, b+c)=f(a, b)+f(a, c)$, by the linearity of $\iota$. Similarly, one has

$$
\begin{aligned}
\tilde{f}(a+b, c) & =s(a+b) \cdot s(c)-s((a+b) \cdot c) \\
& =(s(a)+s(b)) \cdot s(c)-s((a \cdot b) \cdot(a \cdot c)) \\
& =(s(a) \cdot s(b)) \cdot(s(a) \cdot s(c))+\tilde{f}(a \cdot b, a \cdot c)-s(a \cdot b) \cdot s(a \cdot c) \\
& =\tilde{f}(a \cdot b, a \cdot c)+(\tilde{f}(a, b)+s(a \cdot b)) \cdot(s(a) \cdot s(c))-s(a \cdot b) \cdot s(a \cdot c) \\
& \stackrel{(1)}{=} \tilde{f}(a \cdot b, a \cdot c)+s(a \cdot b) \cdot(s(a) \cdot s(c))-s(a \cdot b) \cdot s(a \cdot c) \\
& =\tilde{f}(a \cdot b, a \cdot c)+s(a \cdot b) \cdot(s(a) \cdot s(c)-s(a \cdot c)) \\
& =\tilde{f}(a \cdot b, a \cdot c)+s(a \cdot b) \cdot \tilde{f}(a, c) \\
& \stackrel{(2)}{=} \tilde{f}(a \cdot b, a \cdot c)+\tilde{f}(a, c) .
\end{aligned}
$$

In (1) we got rid of $\tilde{f}(a, b) \in \iota(\Gamma)$ since the centrality of $\iota(\Gamma)$ yields

$$
(\tilde{f}(a, b)+x) \cdot y=(\tilde{f}(a, b) \cdot x) \cdot(\tilde{f}(a, b) \cdot y)=x \cdot y
$$

for all $x, y \in E$. This centrality was also used in (2). The relation $f(a+b, c)=$ $f(a \cdot b, a \cdot c)+f(a, c)$ is now obtained from the corresponding relation for $\tilde{f}$ by applying $\iota^{-1}$.

We will next show that the linear map $\varphi: \Gamma \oplus_{f} A \rightarrow E, \gamma \oplus a \mapsto \iota(\gamma)+s(a)$ yields an equivalence of extensions. It is bijective, the inverse given by the map $x \mapsto \iota^{-1}(x-s \pi(x)) \oplus \pi(x)$ (this map is well defined since $x-s \pi(x) \in \operatorname{Ker} \pi=\operatorname{Im} \iota$ ). Let us check that $\varphi$ intertwines the cycle set operations. One has

$$
\begin{aligned}
\varphi\left((\gamma \oplus a) \cdot\left(\gamma^{\prime} \oplus a^{\prime}\right)\right) & =\varphi\left(\left(\gamma^{\prime}+f\left(a, a^{\prime}\right)\right) \oplus a \cdot a^{\prime}\right)=\iota\left(\gamma^{\prime}+f\left(a, a^{\prime}\right)\right)+s\left(a \cdot a^{\prime}\right) \\
& =\iota\left(\gamma^{\prime}\right)+\tilde{f}\left(a, a^{\prime}\right)+\left(s(a) \cdot s\left(a^{\prime}\right)-\tilde{f}\left(a, a^{\prime}\right)\right) \\
& =\iota\left(\gamma^{\prime}\right)+s(a) \cdot s\left(a^{\prime}\right)=s(a) \cdot \iota\left(\gamma^{\prime}\right)+s(a) \cdot s\left(a^{\prime}\right) \\
& =s(a) \cdot\left(\iota\left(\gamma^{\prime}\right)+s\left(a^{\prime}\right)\right)=(\iota(\gamma)+s(a)) \cdot\left(\iota\left(\gamma^{\prime}\right)+s\left(a^{\prime}\right)\right) \\
& =\varphi(\gamma \oplus a) \cdot \varphi\left(\gamma^{\prime} \oplus a^{\prime}\right) .
\end{aligned}
$$

We use the centrality of $\iota\left(\gamma^{\prime}\right)$ and $\iota(\gamma)$. The commutativity of the diagram (3-5) is obvious, and completes the proof.

Suppose now that the reduced cocycles $f$ and $f^{\prime}$ are obtained from the sections $s$ and $s^{\prime}$ respectively. Put $\tilde{\theta}=s-s^{\prime}: A \rightarrow E$. This is a linear map with its image 
contained in $\operatorname{Ker} \pi=\operatorname{Im} \iota$. Hence it defines a linear map $\theta: A \rightarrow \Gamma$. To show that $f$ and $f^{\prime}$ are cohomologous, we establish the property $f^{\prime}-f=\partial^{1} \theta$ by computing

$$
\begin{aligned}
\left(\tilde{f}-\tilde{f}^{\prime}\right)\left(a, a^{\prime}\right) & =\tilde{f}\left(a, a^{\prime}\right)-\tilde{f}^{\prime}\left(a, a^{\prime}\right) \\
& =s(a) \cdot s\left(a^{\prime}\right)-s\left(a \cdot a^{\prime}\right)-s^{\prime}(a) \cdot s^{\prime}\left(a^{\prime}\right)+s^{\prime}\left(a \cdot a^{\prime}\right) \\
& =s(a) \cdot s\left(a^{\prime}\right)-s(a) \cdot s^{\prime}\left(a^{\prime}\right)-\tilde{\theta}\left(a \cdot a^{\prime}\right) \\
& =s(a) \cdot\left(s\left(a^{\prime}\right)-s^{\prime}\left(a^{\prime}\right)\right)-\tilde{\theta}\left(a \cdot a^{\prime}\right) \\
& =s(a) \cdot \tilde{\theta}\left(a^{\prime}\right)-\tilde{\theta}\left(a \cdot a^{\prime}\right) \stackrel{(1)}{=} \tilde{\theta}\left(a^{\prime}\right)-\tilde{\theta}\left(a \cdot a^{\prime}\right),
\end{aligned}
$$

and applying $\iota^{-1}$, where we use the centrality of $\tilde{\theta}\left(a^{\prime}\right)$ in (1).

We now compare extensions constructed out of different 2-cocycles.

Lemma 3.10. Let $(A, \cdot,+)$ be a linear cycle set, let $\Gamma$ be an abelian group, and let $f, f^{\prime} \in R Z^{2}(A ; \Gamma)$ be two reduced 2-cocycles. The linear cycle set extensions $\Gamma \oplus_{f} A$ and $\Gamma \oplus_{f^{\prime}} A$ are equivalent if and only if $f$ and $f^{\prime}$ are cohomologous.

Proof. Suppose that a linear map $\varphi: \Gamma \oplus_{f} A \rightarrow \Gamma \oplus_{f^{\prime}} A$ provides an equivalence of extensions. The commutativity of the diagram (3-5) forces it to be of the form $\varphi(\gamma \oplus a)=(\gamma+\theta(a)) \oplus a$ for some linear map $\theta: A \rightarrow \Gamma$. Further, one computes

$$
\begin{aligned}
\varphi\left((\gamma \oplus a) \cdot\left(\gamma^{\prime} \oplus a^{\prime}\right)\right) & =\varphi\left(\left(\gamma^{\prime}+f\left(a, a^{\prime}\right)\right) \oplus a \cdot a^{\prime}\right) \\
& =\left(\gamma^{\prime}+f\left(a, a^{\prime}\right)+\theta\left(a \cdot a^{\prime}\right)\right) \oplus a \cdot a^{\prime}, \\
\varphi(\gamma \oplus a) \cdot \varphi\left(\gamma^{\prime} \oplus a^{\prime}\right) & =((\gamma+\theta(a)) \oplus a) \cdot\left(\left(\gamma^{\prime}+\theta\left(a^{\prime}\right)\right) \oplus a^{\prime}\right) \\
& =\left(\gamma^{\prime}+\theta\left(a^{\prime}\right)+f^{\prime}\left(a, a^{\prime}\right)\right) \oplus a \cdot a^{\prime} .
\end{aligned}
$$

Thus the map $\varphi$ entwines the cycle set operations if and only if $f^{\prime}-f$ is the coboundary $\partial^{1} \theta$.

In the opposite direction, take cohomologous cocycles $f$ and $f^{\prime}$. This means that the relation $f^{\prime}-f=\partial^{1} \theta$ holds for a linear map $\theta: A \rightarrow \Gamma$. Repeating the arguments above, one verifies that the map $\varphi(\gamma \oplus a)=(\gamma+\theta(a)) \oplus a$ is an equivalence of extensions $\Gamma \oplus_{f} A \rightarrow \Gamma \oplus_{f^{\prime}} A$.

Put together, the preceding lemmas yield:

Theorem 3.11. Let $(A, \cdot,+)$ be a linear cycle set and $\Gamma$ be an abelian group. The construction from Lemma 3.9 yields a bijective correspondence

$$
\operatorname{CTExt}(A, \Gamma) \stackrel{1: 1}{\longleftrightarrow} R H^{2}(A ; \Gamma)
$$

We finish this section by observing that in degree 2 , the normalization brings nothing new to the reduced LCS cohomology theory: 
Proposition 3.12. In a linear cycle set $(A, \cdot,+)$, every reduced 2-cocycle is normalized. Moreover, one has an isomorphism in cohomology:

$$
R H^{2}(A ; \Gamma) \cong R H_{\mathrm{N}}^{2}(A ; \Gamma) \text {. }
$$

Proof. Putting $a=b=0$ in the defining relation (3-2) for a reduced 2-cocycle $f$, and using the properties of the element 0 from Lemma 2.2, $f(0, c)=0$ for all $c \in A$. Moreover, $f(c, 0)=0$ by linearity. So $f$ is normalized, hence the identification

$$
R Z^{2}(A ; \Gamma)=R Z_{\mathrm{N}}^{2}(A ; \Gamma) .
$$

In degree 1 the normalized and usual complexes coincide, yielding the desired cohomology group isomorphism in degree 2.

\section{Full linear cycle set cohomology}

The previous section treated linear cycle set extensions of the form $\Gamma \oplus_{f} A$. They can be thought of as the direct product $\Gamma \oplus A$ of LCS with the cycle set operation . deformed by $f$. From now on we will handle a more general situation: the additive operation + on $\Gamma \oplus A$ will be deformed as well. Most proofs in this general case are analogous to but more technical than those from the previous sections.

Take a linear cycle set $(A, \cdot,+)$ and an abelian group $\Gamma$. For $i \geqslant 0, j \geqslant 1$, let $\operatorname{ShC}_{i, j}(A ; \Gamma)$ be the abelian subgroup of $\Gamma^{\left(A^{\times(i+j)}\right)}$, generated by the partial shuffles

$$
\sum_{\sigma \in \mathrm{Sh}_{r, j-r}}(-1)^{\sigma} \gamma\left(a_{1}, \ldots, a_{i}, a_{i+\sigma^{-1}(1)}, \ldots, a_{i+\sigma^{-1}(j)}\right)
$$

taken for all $1 \leqslant r \leqslant j-1, a_{k} \in A, \gamma \in \Gamma$. Here $\mathrm{Sh}_{r, j-r}$ is the subset of all the permutations $\sigma$ of $j$ elements satisfying $\sigma(1) \leqslant \cdots \leqslant \sigma(r), \sigma(r+1) \leqslant \cdots \leqslant \sigma(j)$. The term shuffle is used when $i=0$. Put

$$
C_{i, j}(A ; \Gamma)=\Gamma^{\left(A^{\times(i+j)}\right)} / \operatorname{ShC}_{i, j}(A ; \Gamma) .
$$

Recall the notation

$$
\begin{aligned}
\partial_{n ; 0}\left(a_{1}, \ldots, a_{n}\right) & =\left(a_{1} \cdot a_{2}, \ldots, a_{1} \cdot a_{n}\right), \\
\partial_{n ; i}\left(a_{1}, \ldots, a_{n}\right) & =\left(a_{1}, \ldots, a_{i}+a_{i+1}, \ldots, a_{n}\right), \quad 1 \leqslant i \leqslant n-1,
\end{aligned}
$$

from the proof of Proposition 2.1, and consider the coordinate omitting maps

$$
\partial_{n ; i}^{\prime}\left(a_{1}, \ldots, a_{n}\right)=\left(a_{1}, \ldots, a_{i-1}, a_{i+1}, \ldots, a_{n}\right), \quad 1 \leqslant i \leqslant n .
$$


Combine (the linearizations of) these maps into what we will show to be horizontal and vertical differentials of a bicomplex:

$$
\begin{gathered}
\partial_{i, j}^{h}=\partial_{i+j ; 0}+\sum_{k=1}^{i-1}(-1)^{k} \partial_{i+j ; k}+(-1)^{i} \partial_{i+j ; i}^{\prime}, \quad i \geqslant 1, j \geqslant 1 ; \\
-\partial_{i, j}^{v}=\partial_{i+j ; i+1}^{\prime}+\sum_{k=1}^{j-1}(-1)^{k} \partial_{i+j ; i+k}+(-1)^{j} \partial_{i+j ; i+j}^{\prime}, \quad i \geqslant 0, j \geqslant 2 .
\end{gathered}
$$

Here the empty sums are zero by convention. As before, $C_{i, j}^{\mathrm{D}}(A ; \Gamma)$ denotes the abelian subgroup of $\Gamma^{\left(A^{\times(i+j)}\right)}$ generated by the degenerate $(i+j)$-tuples, and $C_{i, j}^{\mathrm{N}}(A ; \Gamma)$ is the quotient $\Gamma^{\left(A^{\times(i+j)}\right)} /\left(C_{i, j}^{\mathrm{D}}(A ; \Gamma)+\operatorname{ShC}_{i, j}(A ; \Gamma)\right)$.

Dually, for $f \in \operatorname{Fun}\left(A^{\times(i+j)}, \Gamma\right)$, put

$$
\partial_{h}^{i, j} f=f \circ \partial_{i+1, j}^{h} \quad \text { and } \quad \partial_{v}^{i, j} f=f \circ \partial_{i, j+1}^{v},
$$

where $i \geqslant 0, j \geqslant 1$, and $f$ is extended to $\mathbb{Z}^{\left(A^{\times(i+j)}\right)}$ by linearity. Let $C^{i, j}(A ; \Gamma)$ be the abelian group of maps $A^{\times(i+j)} \rightarrow \Gamma$ whose linearization vanishes on all partial shuffles (4-1) (with $\gamma$ omitted), and let $C_{\mathrm{N}}^{i, j}(A ; \Gamma) \subseteq C^{i, j}(A ; \Gamma)$ comprise the maps which are moreover zero on all the degenerate $(i+j)$-tuples.

We now assemble these data into both chain and cochain bicomplex structures with normalization.

Theorem 4.1. Let $(A, \cdot,+)$ be a linear cycle set and $\Gamma$ be an abelian group.

(1) The abelian groups $\Gamma^{\left(A^{\times(i+j)}\right)}, i \geqslant 0, j \geqslant 1$, together with the linear maps $\partial_{i, j}^{h}$ and $\partial_{i, j}^{v}$ above, form a chain bicomplex. In other words, the following relations are satisfied:

$$
\begin{array}{ll}
\partial_{i-1, j}^{h} \partial_{i, j}^{h}=0, & i \geqslant 2, j \geqslant 1 ; \\
\partial_{i, j-1}^{v} \partial_{i, j}^{v}=0, & i \geqslant 0, j \geqslant 3 ; \\
\partial_{i, j-1}^{h} \partial_{i, j}^{v}=\partial_{i-1, j}^{v} \partial_{i, j}^{h}, & i \geqslant 1, j \geqslant 2 .
\end{array}
$$

Moreover, these maps restrict to the subgroups $\operatorname{ShC}_{i, j}(A ; \Gamma)$ and $C_{i, j}^{\mathrm{D}}(A ; \Gamma)$, and thus induce chain bicomplex structures on $C_{i, j}(A ; \Gamma)$ and $C_{i, j}^{\mathrm{N}}(A ; \Gamma)$.

(2) The linear maps $\partial_{h}^{i, j}$ and $\partial_{v}^{i, j}$ yield a cochain bicomplex structure for the abelian groups $\operatorname{Fun}\left(A^{\times(i+j)}, \Gamma\right), i \geqslant 0, j \geqslant 1$. This structure restricts to $C^{i, j}(A ; \Gamma)$ and further to $C_{\mathrm{N}}^{i, j}(A ; \Gamma)$.

We abusively denote the induced or restricted maps from the theorem by the same symbols $\partial_{\bullet}^{h}, \partial_{\bullet}^{v}$, etc.

The proof of the theorem relies on the following interpretation of our bicomplex. Its $j$-th row is almost the complex from Proposition 2.1, with a slight modification: 
the last entry in an $n$-tuple, to which the $\partial_{n ; i}$ with $i>0$ did nothing and on which $\partial_{n ; 0}$ acted by a left translation $a_{n} \mapsto a_{1} \cdot a_{n}$, is replaced with the $j$-tuple of last elements behaving in the same way. In the $i$-th column, the first $i$ entries of $A^{\times(i+\bullet)}$ are never affected; on the remaining entries the vertical differentials $\partial_{i, \bullet}^{v}$ act as the differentials from Proposition 2.1 computed for the trivial cycle set operation $a \cdot b=b$. Alternatively, the $i$-th column can be seen as the Hochschild complex for $(A,+)$ with coefficients in $A^{\times i}$, on which $A$ acts trivially on both sides. Modding out $\operatorname{ShC}_{i, j}(A ; \Gamma)$ means passing from the Hochschild to the Harrison complex in each column.

Proof. As usual, it suffices to treat only the homological statements.

Due to the observation preceding the proof, the horizontal relation (4-7) and the vertical relation (4-8) follow from Proposition 2.1. For the mixed relation (4-9), note that the horizontal and vertical differentials involved affect, respectively, the first $i$ and the last $j$ entries of an $(i+j)$-tuple, with the exception of the $\partial_{n ; 0}$ component of $\partial^{h}$. However, this component also commutes with $\partial^{v}$ because of the linearity (with respect to + ) of the left translation $a_{1} \cdot-$ involved.

Applying a left translation $a \cdot-$ to each entry of a partial shuffle (4-1), one still gets a partial shuffle. Consequently, the horizontal differentials $\partial^{h}$ restrict to $\operatorname{ShC}_{i, j}(A ; \Gamma)$. In order to show that the $\partial^{v}$ restrict to $\operatorname{ShC}_{i, j}(A ; \Gamma)$ as well, it suffices to check that the expression

$$
\begin{aligned}
& \sum_{\sigma \in \mathrm{Sh}_{r, j-r}}(-1)^{\sigma}\left(a_{\sigma^{-1}(2)}, \ldots, a_{\sigma^{-1}(j)}\right) \\
&+\sum_{k=1}^{j-1}(-1)^{k} \sum_{\sigma \in \mathrm{Sh}_{r, j-r}}(-1)^{\sigma}\left(a_{\sigma^{-1}(1)}, \ldots, a_{\sigma^{-1}(k)}+a_{\sigma^{-1}(k+1)}, \ldots, a_{\sigma^{-1}(j)}\right) \\
&+(-1)^{j} \sum_{\sigma \in \mathrm{Sh}_{r, j-r}}(-1)^{\sigma}\left(a_{\sigma^{-1}(1)}, \ldots, a_{\sigma^{-1}(j-1)}\right)
\end{aligned}
$$

is a linear combination of shuffles for all $j \geqslant 1,1 \leqslant r \leqslant j-1, a_{k} \in A$. Let $S_{1}, S_{2}$, and $S_{3}$ denote the three sums above, and consider the classical notation

$$
\uplus_{r, j-r}\left(a_{1}, \ldots, a_{j}\right)=\sum_{\sigma \in \mathrm{Sh}_{r, j-r}}(-1)^{\sigma}\left(a_{\sigma^{-1}(1)}, \ldots, a_{\sigma^{-1}(j)}\right)
$$

for shuffles, and the convention $\sqcup_{0, j}=\uplus_{j, 0}=$ Id. Recall also notation (4-3)-(4-4). The sums $S_{i}$ then rewrite as

$$
\begin{aligned}
& S_{1}=\uplus_{r-1, j-r} \partial_{j ; 1}^{\prime}+(-1)^{r} \uplus_{r, j-r-1} \partial_{j ; r+1}^{\prime}, \\
& S_{3}=(-1)^{r} \uplus_{r-1, j-r} \partial_{j ; r}^{\prime}+(-1)^{j} \uplus_{r, j-r-1} \partial_{j ; j}^{\prime}, \\
& S_{2}=\sum_{k=1}^{r-1}(-1)^{k} \uplus_{r-1, j-r} \partial_{j ; k}+\sum_{k=r+1}^{j-1}(-1)^{k} \uplus_{r, j-r-1} \partial_{j ; k},
\end{aligned}
$$


with empty sums declared to be zero. The decomposition for $S_{1}$ follows from the analysis of the two possibilities for $\sigma^{-1}(1)$ with $\sigma \in \mathrm{Sh}_{r, j-r}$, namely, $\sigma^{-1}(1)=1$ and $\sigma^{-1}(1)=r+1$. The decomposition for $S_{3}$ corresponds to the dichotomy $\sigma^{-1}(j)=r$ or $\sigma^{-1}(j)=j$. In $S_{3}$, the summands with $\sigma^{-1}(k)=u \leqslant r<v=\sigma^{-1}(k+1)$ and $\sigma^{-1}(k)=v, \sigma^{-1}(k+1)=u$ appear with opposite signs and can therefore be discarded. The remaining ones can be divided into two classes: those with $\sigma^{-1}(k)<\sigma^{-1}(k+1) \leqslant r$ and those with $r<\sigma^{-1}(k)<\sigma^{-1}(k+1)$, giving the decomposition above. Our $S_{i}$ are thus signed sums of shuffles, with the exception of the cases $r \in\{1, j-1\}$. For $r=1$, the nonshuffle terms $\partial_{j ; 1}^{\prime}$ and $-\partial_{j ; 1}^{\prime}$ appear in $S_{1}$ and $S_{3}$ respectively; they annihilate each other in the total sum. The case $r=j-1$ is treated similarly.

The possibility to restrict all the $\partial^{h}$ and $\partial^{v}$ to $C_{i, j}^{\mathrm{D}}(A ; \Gamma)$ is taken care of, as usual, by Lemma 2.2. As a consequence, one obtains a chain bicomplex structure on $C_{i, j}^{\mathrm{N}}(A ; \Gamma)$.

We are now in a position to define the full (co)homology of a linear cycle set:

Definition 4.2. The cycles, boundaries, homology groups of a linear cycle set $(A, \cdot,+)$ with coefficients in an abelian group $\Gamma$ are those of the total chain complex

$$
\left(C_{n}(A ; \Gamma)=\bigoplus_{i+j=n} C_{i, j}(A ; \Gamma),\left.\partial_{n}\right|_{C_{i, j}}=\partial_{i, j}^{h}+(-1)^{i} \partial_{i, j}^{v}\right)
$$

of the bicomplex above. Dually, the cocycles, coboundaries, cohomology groups of $(A, \cdot,+)$ are those of the complex

$$
\left(C^{n}(A ; \Gamma)=\bigoplus_{i+j=n} C^{i, j}(A ; \Gamma), \partial^{n}=\partial_{n+1}^{*}\right) .
$$

In the normalized case, one uses the complexes

$$
\left(C_{n}^{\mathrm{N}}(A ; \Gamma)=\bigoplus_{i+j=n} C_{i, j}^{\mathrm{N}}(A ; \Gamma), \partial_{n}\right) \quad \text { and } \quad\left(C_{\mathrm{N}}^{n}(A ; \Gamma)=\bigoplus_{i+j=n} C_{\mathrm{N}}^{i, j}(A ; \Gamma), \partial^{n}\right) .
$$

We use the usual notations $Q_{n}(A ; \Gamma)$, etc., where $Q$ is one of the letters $Z, B$, or $H$.

Remark 4.3. In fact our (co)chain bicomplex constructions can be refined into bisimplicial ones.

Remark 4.4. Instead of considering the total complex of our bicomplex, one could start by, say, computing the homology $H_{i, \bullet}^{v}$ of each column. The horizontal differentials then induce a chain complex structure on each row $H_{\bullet, j}^{v}$. Observe that the first row is precisely the complex from Proposition 2.1. Its homology is then the reduced homology of our linear cycle set. 


\section{General linear cycle set extensions}

Our next step is to describe what a 2-cocycle looks like for the full version of linear cycle set cohomology theory. Such a 2-cocycle consists of two components $f, g: A \times A \rightarrow \Gamma$, seen as elements of $C^{1,1}(A ; \Gamma)=\operatorname{Fun}(A \times A, \Gamma)$ and $C^{0,2}(A ; \Gamma)=$ $\operatorname{Sym}(A \times A, \Gamma)$, respectively. Here Sym denotes the abelian group of symmetric maps, i.e., satisfying

$$
g(a, b)=g(b, a) .
$$

These maps should satisfy three identities, one for each component of

$$
C^{3}(A ; \Gamma)=C^{2,1}(A ; \Gamma) \oplus C^{1,2}(A ; \Gamma) \oplus C^{0,3}(A ; \Gamma) .
$$

Explicitly, these identities read

$$
\begin{aligned}
f(a+b, c) & =f(a \cdot b, a \cdot c)+f(a, c), \\
f(a, b+c)-f(a, b)-f(a, c) & =g(a \cdot b, a \cdot c)-g(b, c), \\
g(a, b)+g(a+b, c) & =g(b, c)+g(a, b+c) .
\end{aligned}
$$

In particular, $f$ is a 2-cocycle of the cycle set $(A, \cdot)$, and $g$ is a symmetric 2-cocycle of the group $(A,+)$. The reduced cocycles are precisely those with $g=0$. Further, the 2-coboundaries are couples of maps

$$
\begin{aligned}
& f(a, b)=\theta(a \cdot b)-\theta(b), \\
& g(a, b)=\theta(a+b)-\theta(a)-\theta(b)
\end{aligned}
$$

for some $\theta: A \rightarrow \Gamma$.

We next give some elementary properties of 2-cocycles and 2-coboundaries.

Lemma 5.1. Let $(f, g)$ be a 2-cocycle of a linear cycle set $(A, \cdot,+)$ with coefficients in an abelian group $\Gamma$.

(1) For all $x \in A$,

$$
\begin{aligned}
& f(0, x)=f(x, 0)=0, \\
& g(0, x)=g(x, 0)=g(0,0) .
\end{aligned}
$$

(2) The 2-cocycle $(f, g)$ is normalized if and only if $g(0,0)=0$.

Proof. Let us prove the first claim. The relation $f(0, x)=0$ follows from (5-2) by choosing $a=0$. Similarly, the relation $f(x, 0)=0$ is (5-3) specialized at $b=c=0$. Substitutions $b=0$ and either $a=0$ or $c=0$ in (5-4) yield the last relation. Now the second claim directly follows from the previous point. 
Lemma 5.2. Let $(A, \cdot,+)$ be a linear cycle set, let $\Gamma$ be an abelian group, and let $f, g: A \times A \rightarrow \Gamma$ be two maps. Then the set $\Gamma \times A$ with the operations

$$
\begin{aligned}
(\gamma, a)+\left(\gamma^{\prime}, a^{\prime}\right) & =\left(\gamma+\gamma^{\prime}+g\left(a, a^{\prime}\right), a+a^{\prime}\right), \\
(\gamma, a) \cdot\left(\gamma^{\prime}, a^{\prime}\right) & =\left(\gamma^{\prime}+f\left(a, a^{\prime}\right), a \cdot a^{\prime}\right)
\end{aligned}
$$

for $\gamma, \gamma^{\prime} \in \Gamma, a, a^{\prime} \in A$, is a linear cycle set if and only if $(f, g)$ is a 2-cocycle, i.e., $(f, g) \in Z^{2}(A ; \Gamma)$.

Notation 5.3. The LCS from the lemma is denoted by $\Gamma \oplus_{f, g} A$.

Proof. The left translation invertibility for $\Gamma \oplus_{f, g} A$ follows from the same property for $A$. Properties (1-3) and (1-4) for $\Gamma \oplus_{f, g} A$ are equivalent to, respectively, properties (5-3) and (5-2) for $(f, g)$. The associativity and the commutativity of + on $\Gamma \oplus_{f, g} A$ are encoded by property (5-4) for $\Gamma \oplus_{f, g} A$ and the symmetry of $g$ respectively. Finally, if $(f, g)$ is a 2-cocycle, then Lemma 5.1 implies that $(-g(0,0), 0)$ is the zero element for $\left(\Gamma \oplus_{f, g} A,+\right)$, and the opposite of $(\gamma, a)$ is $(-\gamma-g(0,0)-g(a,-a),-a)$.

As we did in Lemma 3.5, we now translate Lemma 5.2 into the language of braces.

Lemma 5.4. Let $(A, \circ,+)$ be a brace, $\Gamma$ be an abelian group, and $f, g: A \times A \rightarrow \Gamma$ be two maps. Then the set $\Gamma \times A$ with the operations

$$
\begin{gathered}
(\gamma, a)+\left(\gamma^{\prime}, a^{\prime}\right)=\left(\gamma+\gamma^{\prime}+g\left(a, a^{\prime}\right), a+a^{\prime}\right), \\
(\gamma, a) \circ\left(\gamma^{\prime}, a^{\prime}\right)=\left(\gamma+\gamma^{\prime}+f\left(a, a^{\prime}\right), a \circ a^{\prime}\right)
\end{gathered}
$$

for $\gamma, \gamma^{\prime} \in \Gamma, a, a^{\prime} \in A$, is a brace if and only if for the corresponding linear cycle set $(A, \cdot,+)$, the maps

$$
\bar{f}(a, b)=-f(a, a \cdot b)+g(a, b)
$$

and $g$ form a 2-cocycle $(\bar{f}, g) \in Z^{2}(A ; \Gamma)$.

Proof. Recall the correspondence $a \cdot b=a^{-1} \circ(a+b)$ between the corresponding brace and LCS operations. It can also be rewritten as $a \circ b=a+a * b$, where the map $a \mapsto a * b$ is the inverse of the left translation $a \mapsto a \cdot b$.

Now, given any $(\bar{f}, g) \in Z^{2}(A ; \Gamma)$, the formulas from Lemma 5.2 describe an LCS structure on $\Gamma \times A$. Its operation $*$ reads

$$
(\gamma, a) *\left(\gamma^{\prime}, a^{\prime}\right)=\left(\gamma^{\prime}-\bar{f}\left(a, a * a^{\prime}\right), a * a^{\prime}\right) .
$$

The operations

$$
(\gamma, a)+\left(\gamma^{\prime}, a^{\prime}\right)=\left(\gamma+\gamma^{\prime}+g\left(a, a^{\prime}\right), a+a^{\prime}\right),
$$


and

$$
\begin{aligned}
(\gamma, a) \circ\left(\gamma^{\prime}, a^{\prime}\right) & =(\gamma, a)+(\gamma, a) *\left(\gamma^{\prime}, a^{\prime}\right) \\
& =\left(\gamma+\gamma^{\prime}-\bar{f}\left(a, a * a^{\prime}\right)+g\left(a, a * a^{\prime}\right), a \circ a^{\prime}\right)
\end{aligned}
$$

then yield a brace structure on $\Gamma \times A$. These formulas have the desired form, with

$$
f\left(a, a^{\prime}\right)=-\bar{f}\left(a, a * a^{\prime}\right)+g\left(a, a * a^{\prime}\right),
$$

which, through the substitution $b=a * a^{\prime}$, is equivalent to (5-7).

Conversely, starting from a brace structure on $\Gamma \times A$ of the desired form, one sees that its associated LCS structure is as described in Lemma 5.2 with some $(\bar{f}, g) \in Z^{2}(A ; \Gamma)$. Repeating the argument above, one obtains the relation $(5-7)$ connecting $f, \bar{f}$, and $g$.

Definition 5.5. A central extension of a linear cycle set $(A, \cdot,+)$ by an abelian group $\Gamma$ is the datum of a short exact sequence of linear cycle sets

$$
0 \rightarrow \Gamma \stackrel{\iota}{\rightarrow} E \stackrel{\pi}{\rightarrow} A \rightarrow 0
$$

where $\Gamma$ is endowed with the trivial cycle set structure, and its image $\iota(\Gamma)$ is central in $E$ (in the sense of Definition 3.6). The notion of equivalence for central cycle-type LCS extensions (Definition 3.8) transports verbatim to these general extensions. The set of equivalence classes of central extensions of $A$ by $\Gamma$ is denoted by $\operatorname{Ext}(A, \Gamma)$.

The LCS $\Gamma \oplus_{f, g} A$ from Lemma 5.2 is an extension of $A$ by $\Gamma$ in the obvious way. We now show that this example is essentially exhaustive.

Lemma 5.6. Let $\Gamma \stackrel{\iota}{\longmapsto} E \stackrel{\pi}{\longrightarrow} A$ be a central LCS extension, and let $s: A \rightarrow E$ be a set-theoretic section of $\pi$.

(1) The maps $\tilde{f}, \tilde{g}: A \times A \rightarrow E$ defined by

$$
\begin{aligned}
& \tilde{f}:\left(a, a^{\prime}\right) \mapsto s(a) \cdot s\left(a^{\prime}\right)-s\left(a \cdot a^{\prime}\right), \\
& \tilde{g}:\left(a, a^{\prime}\right) \mapsto s(a)+s\left(a^{\prime}\right)-s\left(a+a^{\prime}\right)
\end{aligned}
$$

both take values in $\iota(\Gamma)$ and determine a cocycle $(f, g) \in Z^{2}(A ; \Gamma)$.

(2) The cocycle above is normalized if and only if $s$ is such, in the sense of $s(0)=0$.

(3) Extensions $E$ and $\Gamma \oplus_{f, g} A$ are equivalent.

(4) A cocycle $\left(f^{\prime}, g^{\prime}\right)$ obtained from another section $s^{\prime}$ of $\pi$ is cohomologous to $(f, g)$. If both cocycles are normalized, then they are cohomologous in the normalized sense.

Lemma 5.7. Let $(A, \cdot,+)$ be a linear cycle set, let $\Gamma$ be an abelian group, and let $(f, g),\left(f^{\prime}, g^{\prime}\right) \in Z^{2}(A ; \Gamma)$ be 2-cocycles. The linear cycle set extensions $\Gamma \oplus_{f, g} A$ and $\Gamma \oplus_{f^{\prime}, g^{\prime}} A$ are equivalent if and only if the cocycle $(f, g)-\left(f^{\prime}, g^{\prime}\right)$ is a normalized 2-coboundary. 
Recall that a normalized 2-coboundary is a couple of maps of the form $\partial^{1} \theta$, where the map $\theta: A \rightarrow \Gamma$ is normalized, in the sense of $\theta(0)=0$.

The proof of these lemmas is technical but conceptually analogous to the proofs of Lemmas 3.9 and 3.10, and will therefore be omitted.

Put together, the preceding lemmas prove:

Theorem 5.8. Let $(A, \cdot,+)$ be a linear cycle set and $\Gamma$ be an abelian group. The construction from Lemma 5.6 yields a bijective correspondence

$$
\operatorname{Ext}(A, \Gamma) \stackrel{1: 1}{\longleftrightarrow} H_{\mathrm{N}}^{2}(A ; \Gamma) .
$$

In other words, the central extensions of LCS (and thus of braces) are completely determined by their second normalized cohomology groups.

\section{Acknowledgments}

The work of L. Vendramin is partially supported by CONICET, PICT-2014-1376, MATH-AmSud, and ICTP. V. Lebed thanks the program ANR-11-LABX-0020-01 and Henri Lebesgue Center (University of Nantes) for support. The authors are grateful to the reviewer for useful remarks and interesting suggestions for a further development of the subject.

\section{References}

[Bachiller 2015a] D. Bachiller, "Classification of braces of order $p^{3 ",}$ J. Pure Appl. Algebra 219:8 (2015), 3568-3603. MR Zbl

[Bachiller 2015b] D. Bachiller, "Examples of simple left braces", preprint, 2015. arXiv

[Bachiller et al. 2015a] D. Bachiller, F. Cedo, and E. Jespers, "Solutions of the Yang-Baxter equation associated with a left brace", preprint, 2015. arXiv

[Bachiller et al. 2015b] D. Bachiller, F. Cedo, E. Jespers, and J. Okninski, "A family of irretractable square-free solutions of the Yang-Baxter equation", preprint, 2015. arXiv

[Ben David and Ginosar 2016] N. Ben David and Y. Ginosar, "On groups of I-type and involutive Yang-Baxter groups", J. Algebra 458 (2016), 197-206. MR

[Catino and Rizzo 2009] F. Catino and R. Rizzo, "Regular subgroups of the affine group and radical circle algebras”, Bull. Aust. Math. Soc. 79:1 (2009), 103-107. MR Zbl

[Catino et al. 2015] F. Catino, I. Colazzo, and P. Stefanelli, "On regular subgroups of the affine group”, Bull. Aust. Math. Soc. 91:1 (2015), 76-85. MR Zbl

[Catino et al. 2016] F. Catino, I. Colazzo, and P. Stefanelli, "Regular subgroups of the affine group and asymmetric product of radical braces”, J. Algebra 455 (2016), 164-182. MR Zbl

[Cedó et al. 2010a] F. Cedó, E. Jespers, and J. Okniński, "Retractability of set theoretic solutions of the Yang-Baxter equation”, Adv. Math. 224:6 (2010), 2472-2484. MR Zbl

[Cedó et al. 2010b] F. Cedó, E. Jespers, and Á. del Río, "Involutive Yang-Baxter groups", Trans. Amer. Math. Soc. 362:5 (2010), 2541-2558. MR Zbl 
[Cedó et al. 2014] F. Cedó, E. Jespers, and J. Okniński, "Braces and the Yang-Baxter equation", Comm. Math. Phys. 327:1 (2014), 101-116. MR Zbl

[Chouraqui 2010] F. Chouraqui, "Garside groups and Yang-Baxter equation", Comm. Algebra 38:12 (2010), 4441-4460. MR Zbl

[Dehornoy 2015] P. Dehornoy, "Set-theoretic solutions of the Yang-Baxter equation, RC-calculus, and Garside germs", Adv. Math. 282 (2015), 93-127. MR Zbl

[Etingof et al. 1999] P. Etingof, T. Schedler, and A. Soloviev, "Set-theoretical solutions to the quantum Yang-Baxter equation”, Duke Math. J. 100:2 (1999), 169-209. MR Zbl

[Gateva-Ivanova 2004] T. Gateva-Ivanova, "A combinatorial approach to the set-theoretic solutions of the Yang-Baxter equation", J. Math. Phys. 45:10 (2004), 3828-3858. MR Zbl

[Gateva-Ivanova 2015] T. Gateva-Ivanova, "Set-theoretic solutions of the Yang-Baxter equation, Braces, and Symmetric groups", preprint, 2015. arXiv

[Gateva-Ivanova and Cameron 2012] T. Gateva-Ivanova and P. Cameron, "Multipermutation solutions of the Yang-Baxter equation”, Comm. Math. Phys. 309:3 (2012), 583-621. MR Zbl

[Gateva-Ivanova and Majid 2008] T. Gateva-Ivanova and S. Majid, "Matched pairs approach to set theoretic solutions of the Yang-Baxter equation”, J. Algebra 319:4 (2008), 1462-1529. MR Zbl

[Gateva-Ivanova and Van den Bergh 1998] T. Gateva-Ivanova and M. Van den Bergh, "Semigroups of I-type", J. Algebra 206:1 (1998), 97-112. MR Zbl

[Jespers and Okniński 2005] E. Jespers and J. Okniński, "Monoids and groups of I-type", Algebr. Represent. Theory 8:5 (2005), 709-729. MR Zbl

[Lebed and Vendramin 2015] V. Lebed and L. Vendramin, "Homology of left non-degenerate settheoretic solutions to the Yang-Baxter equation", preprint, 2015. arXiv

[Lu et al. 2000] J.-H. Lu, M. Yan, and Y.-C. Zhu, "On the set-theoretical Yang-Baxter equation", Duke Math. J. 104:1 (2000), 1-18. MR Zbl

[Rump 2005] W. Rump, "A decomposition theorem for square-free unitary solutions of the quantum Yang-Baxter equation”, Adv. Math. 193:1 (2005), 40-55. MR Zbl

[Rump 2007] W. Rump, "Braces, radical rings, and the quantum Yang-Baxter equation", J. Algebra 307:1 (2007), 153-170. MR Zbl

[Rump 2008] W. Rump, "Semidirect products in algebraic logic and solutions of the quantum Yang-Baxter equation”, J. Algebra Appl. 7:4 (2008), 471-490. MR Zbl

[Rump 2014] W. Rump, “The brace of a classical group”, Note Mat. 34:1 (2014), 115-144. MR Zbl

[Smoktunowicz 2015a] A. Smoktunowicz, "A note on set-theoretic solutions of the Yang-Baxter equation", preprint, 2015. arXiv

[Smoktunowicz 2015b] A. Smoktunowicz, "On Engel groups, nilpotent groups, rings, braces and the Yang-Baxter equation”, preprint, 2015. arXiv

[Soloviev 2000] A. Soloviev, "Non-unitary set-theoretical solutions to the quantum Yang-Baxter equation", Math. Res. Lett. 7:5-6 (2000), 577-596. MR Zbl

[Vendramin 2016] L. Vendramin, "Extensions of set-theoretic solutions of the Yang-Baxter equation and a conjecture of Gateva-Ivanova”, J. Pure Appl. Algebra 220:5 (2016), 2064-2076. MR Zbl

Received February 2, 2016. Revised March 22, 2016. 
VICTORIA LEBED

LABORATOIRE DE MATHÉMATIQUES JEAN LERAY

UNIVERSITÉ DE NANTES

2 RUE DE LA HOUSSINIÈRE

BP 92208

44322 Nantes Cedex 3

FRANCE

victoria.lebed@univ-nantes.fr

LEANDRo VENDRAMIN

Departamento de Matemática, FCEN

UNIVERSIDAD DE BUENOS AIRES

PABELLÓN 1

1428 Buenos Aires

ARGENTINA

lvendramin@dm.uba.ar 


\title{
PACIFIC JOURNAL OF MATHEMATICS
}

Founded in 1951 by E. F. Beckenbach (1906-1982) and F. Wolf (1904-1989)

$$
\text { msp.org/pjm }
$$

\section{EDITORS}

\author{
Don Blasius (Managing Editor) \\ Department of Mathematics \\ University of California \\ Los Angeles, CA 90095-1555 \\ blasius@math.ucla.edu
}

\author{
Paul Balmer \\ Department of Mathematics \\ University of California \\ Los Angeles, CA 90095-1555 \\ balmer@math.ucla.edu \\ Robert Finn \\ Department of Mathematics \\ Stanford University \\ Stanford, CA 94305-2125 \\ finn@math.stanford.edu \\ Sorin Popa \\ Department of Mathematics \\ University of California \\ Los Angeles, CA 90095-1555 \\ popa@math.ucla.edu
}

\author{
Vyjayanthi Chari \\ Department of Mathematics \\ University of California \\ Riverside, CA 92521-0135 \\ chari@math.ucr.edu \\ Kefeng Liu \\ Department of Mathematics \\ University of California \\ Los Angeles, CA 90095-1555 \\ liu@math.ucla.edu \\ Igor Pak \\ Department of Mathematics \\ University of California \\ Los Angeles, CA 90095-1555 \\ pak.pjm@gmail.com \\ Paul Yang \\ Department of Mathematics \\ Princeton University \\ Princeton NJ 08544-1000 \\ yang@math.princeton.edu
}

\section{PRODUCTION}

Silvio Levy, Scientific Editor, production@msp.org

\section{SUPPORTING INSTITUTIONS}

ACADEMIA SINICA, TAIPEI

CALIFORNIA INST. OF TECHNOLOGY

STANFORD UNIVERSITY

UNIV. OF BRITISH COLUMBIA

UNIV. OF CALIFORNIA, BERKELEY

UNIV. OF CALIFORNIA, DAVIS

UNIV. OF CALIFORNIA, LOS ANGELES

UNIV. OF CALIFORNIA, RIVERSIDE

UNIV. OF CALIFORNIA, SAN DIEGO

UNIV. OF CALIF., SANTA BARBARA
KEIO UNIVERSITY

MATH. SCIENCES RESEARCH INSTITUTE

NEW MEXICO STATE UNIV.

OREGON STATE UNIV.
Daryl Cooper

Department of Mathematics

University of California

Santa Barbara, CA 93106-3080 cooper@math.ucsb.edu

Jiang-Hua Lu

Department of Mathematics

The University of Hong Kong

Pokfulam Rd., Hong Kong

jhlu@maths.hku.hk

$$
\text { Jie Qing }
$$

Department of Mathematics

University of California

Santa Cruz, CA 95064

qing@ cats.ucsc.edu

\author{
UNIV. OF CALIF., SANTA CRUZ \\ UNIV. OF MONTANA \\ UNIV. OF OREGON \\ UNIV. OF SOUTHERN CALIFORNIA \\ UNIV. OF UTAH \\ UNIV. OF WASHINGTON \\ WASHINGTON STATE UNIVERSITY
}

These supporting institutions contribute to the cost of publication of this Journal, but they are not owners or publishers and have no responsibility for its contents or policies.

See inside back cover or msp.org/pjm for submission instructions.

The subscription price for 2016 is US $\$ 440 /$ year for the electronic version, and \$600/year for print and electronic.

Subscriptions, requests for back issues and changes of subscriber address should be sent to Pacific Journal of Mathematics, P.O. Box 4163, Berkeley, CA 94704-0163, U.S.A. The Pacific Journal of Mathematics is indexed by Mathematical Reviews, Zentralblatt MATH, PASCAL CNRS Index, Referativnyi Zhurnal, Current Mathematical Publications and Web of Knowledge (Science Citation Index).

The Pacific Journal of Mathematics (ISSN 0030-8730) at the University of California, c/o Department of Mathematics, 798 Evans Hall \#3840, Berkeley, CA 94720-3840, is published twelve times a year. Periodical rate postage paid at Berkeley, CA 94704, and additional mailing offices. POSTMASTER: send address changes to Pacific Journal of Mathematics, P.O. Box 4163, Berkeley, CA 94704-0163.

PJM peer review and production are managed by EditFLOW ${ }^{\circledR}$ from Mathematical Sciences Publishers.

PUBLISHED BY

\section{I. mathematical sciences publishers}

nonprofit scientific publishing

http://msp.org/

(C) 2016 Mathematical Sciences Publishers 


\section{PACIFIC JOURNAL OF MATHEMATICS}

Volume $284 \quad$ No. $1 \quad$ September 2016

Bitwist manifolds and two-bridge knots

JAmes W. CANNON, William J. Floyd, LEeR LAMbert,

WALTER R. PARry and Jessica S. PurCELL

Recognizing right-angled Coxeter groups using involutions

Charles Cunningham, Andy Eisenberg, Adam Piggott and KIM RUANE

On Yamabe-type problems on Riemannian manifolds with boundary

Marco Ghimenti, Anna Maria Micheletti and Angela

PISTOIA

Quantifying separability in virtually special groups

MARK F. HAGEN and PRIYAM PATEL

Conformal designs and minimal conformal weight spaces of vertex operator superalgebras

TOMONORI HASHIKAWA

Coaction functors

S. KALiszewski, Magnus B. LANDSTAD and John QuigG

Cohomology and extensions of braces

VICTORIA LEBED and LEANDRO VENDRAMIN

Noncommutative differentials on Poisson-Lie groups and pre-Lie algebras

SHAHN MAJID and WEN-QING TAO 